\title{
Numbering-up and mass transfer studies of liquid-liquid two-phase microstructured reactors
}

\author{
M.N. Kashid*, A. Gupta, A. Renken, L. Kiwi-Minsker \\ Group of Catalytic Reaction Engineering (GGRC), Ecole Polytechnique Fédérale de Lausanne (EPFL), Station 6, CH - 1015 Lausanne, Switzerland
}

\section{A R T I C L E I N F O}

\section{Article history:}

Received 16 September 2009

Received in revised form 6 January 2010

Accepted 11 January 2010

\section{Keywords:}

Microstructured reactors

Liquid-liquid slug-flow

Numbering-up

Mass transfer

Process intensification

\begin{abstract}
A B S T R A C T
Microstructured reactors are known to provide high heat and mass transfer rates for liquid-liquid twophase systems when used as a single channel. For industrial scale production, scale-up of microstructured reactors is essential to achieve throughput in the required range which is done by numbering-up of a single channel. The important issues are uniform flow distribution and identical slug size in all channels. In the present work, a capillary microstructured reactor is numbered up for six capillaries and the mass transfer performance is investigated for various operating conditions. A cationic surfactant was used to study the effect of interfacial tension on the mass transfer performance. If compared to conventional contactors, the mass transfer coefficients were approximately one order of magnitude higher allowing the process intensification. The results obtained demonstrate the benefits of microstructured reactors and confirm that the throughput of conventional reactors can be achieved.
\end{abstract}

(C) 2010 Elsevier B.V. All rights reserved.

\section{Introduction}

Liquid-liquid systems are of huge importance in different chemical processes such as solvent extraction, reactive extraction, nitration, polymerization, and phase transfer catalysis. Often these processes are limited by heat and mass transfer. Microstructured reactors (MSR) offer several processing advantages due to high specific interfacial area improving heat and mass transfer [1]. Two stable flow regimes are possible in the MSR with two immiscible fluids: slug-flow and parallel flow. The enhanced performance is observed in the slug-flow due to two mass transport mechanisms: convection through the internal circulation within each slug and diffusion between adjacent slugs. The shear between the capillary wall and slug axis generates intense internal circulations within the slug, which in turn reduces the thickness of interfacial boundary layer and thereby augments the diffusive penetration.

There have been studies on liquid-liquid two-phase slug-flow [2-6] in which hydrodynamics and mass transfer aspects have been studied. Reviews on different types of liquid-liquid twophase MSR are available in the literature [7,8]. Parallel flow and slug-flow form a well-defined environment for mass transfer during multiphase chemical reactions. A liquid-liquid slug-flow MSR was developed for extraction of acetic acid from kerosene slugs into water by Burns and Ramshaw [2]. The same reactor concept

\footnotetext{
* Corresponding author. Tel.: +41 216933 176; fax: +41 216936091.

E-mail address: madhvanand.kashid@epfl.ch (M.N. Kashid).
}

was further employed for nitration reaction and reported high apparent reaction rates $[1,9]$. Further, the mass transfer performance of different types of MSR for non-reacting [4,5] and reacting [6] systems was investigated and reported 2-3 orders of magnitude higher mass transfer rates compared to their conventional counterparts.

From the above literature review, it is clear that all studies are confined to single channel. For industrial scale production, scaleup of MSR is essential to achieve throughput in the required range. In MSR, scale-up is done by numbering-up or scaling-out, i.e. the multiple, parallel repetitions of micro-processing units. When one considers the attention that has been lavished on studies in single capillary, the paucity of publications on numbering-up studies is somewhat surprising. As per flow distribution is concerned, Schenk et al. [10] reported development of liquid-flow splitting unit for flow distribution of single phase in the range required for MSR. Further, Iwasaki et al. [11] constructed a microchemical pilot plant consisting of a T-shaped micromixer (ID $250 \mu \mathrm{m}$ ) and a shell, and tube numbering-up reactor for radical polymerization of methyl methacrylate (MMA).

Since most of the MSR operate in laminar flow regime, the low velocities show well-defined flow patterns. On the other hand, to create fine dispersion for high interfacial area in two-phase system, the conventional reactors operate under turbulent flow regime and often lead to non-uniform drop size limiting control of their performance. Though the MSR overcomes this limitation, its performance in some applications is limited due to shorter residence time. Therefore, if the laminar flow in MSR is combined with mass transfer enhancing media, then it would be a 
powerful equipment for liquid-liquid operation. Ionic surfactants have been used advantageously to enhance the mass transfer in ternary systems [12]. Thus, two topics are considered in the present work: numbering-up of the MSR and the effect of surfactant on the mass transfer performance. Though the reaction was not considered in the present work, the generic term "reactor" instead of "extractor" or "contactor" was used due to its wide range of applications such as extraction, reactive extraction, chemical reaction, etc. A numbered-up experimental set-up was developed and flow distribution was characterized. The mass transfer performance for different operating conditions varying flow velocity and surfactant concentration was also investigated. The results are compared with the single capillary experiments done previously $[3,4]$ in order to evaluate the performance of numbered setup.

\section{Numbering-up concepts for two-phase MSR}

Numbering-up is preferred over the conventional scale-up because the performance of MSR can be preserved and operational capacity of the industrial reactor can be achieved. In the case of single phase flow, flow distribution with minimum energy consumption is the only key issue in the numbering-up for high throughput. However, in two-phase slug-flow, in addition to flow distribution, the slug size should be uniform in all parallel channels as it affects the performance of the MSR. As mentioned before, there are two ways to number-up MSR for two-phase reactions (Fig. 1): internal numbering-up and external numbering-up. The main functional element of the experimental set-up is numberedup in former case while the whole laboratory set-up is replicated in the latter case. The advantages and limitations of both approaches are explained in the following subsection.

\subsection{Internal numbering-up}

The scheme of internal numbering-up is shown in Fig. 1a. As can be seen, two liquids are mixed in the mixing zone and the biphasic mixture is distributed in several parallel microchannels. The advantage is that it reduces the cost associated with the pumping and mixing equipments as only two pumps and one mixing element is used. However, the distribution of biphasic mixture into parallel channel is a challenging task.

To distribute fluid in parallel channels, equal pressure drop in all channels is required. In the case of single phase flow, the pressure drop is created either at the outlet or at the inlet of the channel using venturi effect which facilitates the uniform flow distribution. However, the pressure drop in the two-phase MSR has additional contribution due to the surface tension, Laplace pressure, which depends on the size of the slug. If the slug size deviates from one channel to the other, the pressure drop also changes leading to nonuniform flow distribution. Further, if the pressure drop is similar in all channels at different slug sizes, the performance of each channel differs from the other due to different interfacial area. A common approach to overcome this problem in microfluidic devices is to use a bigger manifold to uniform the pressure across all channel inlets. However, such a manifold can deviate the MSR performance from the plug flow and the advantages of single channel MSR can be vanished. Another method is to put obstacles at the channel inlets to increase the pressure in the distributor zone but such an approach also results in misdistribution due to two-phase flow.

\subsection{External numbering-up}

The schematic of external numbering-up is depicted in Fig. 1b. It shows that two fluids are introduced to the mixing element of two-phase MSR which are arranged in parallel. The arrangement shows that the laboratory set-up is replicated depending on the required throughput. The advantage is that the performance of single MSR can be preserved in a high throughput facility. However, higher equipment cost and larger set-up size limits the use of this concept.

Thus, two points can be highlighted from the above discussion: precise distribution of single phase liquid is possible and performance of MSR can be achieved in numbered-up setup if each MSR gets a separate two-fluid mixing element. Thus, in this work we combine the above advantages and use an integrated approach: internal numbering-up for distributing single phase fluids and external numbering-up for two-phase contacting. This will not only reduce the cost of the equipment but also its size.

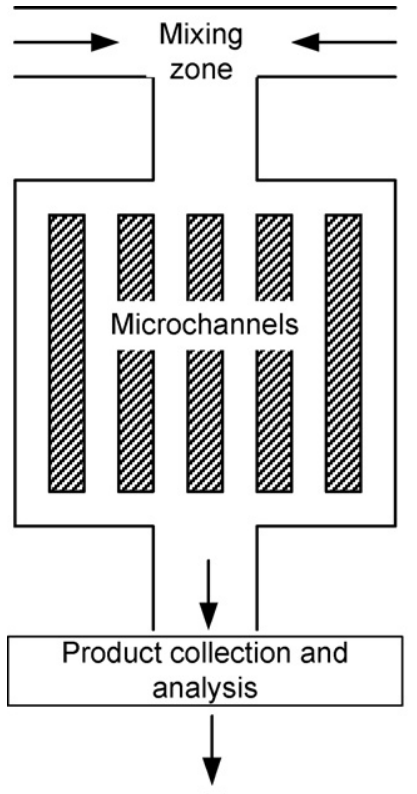

(a)

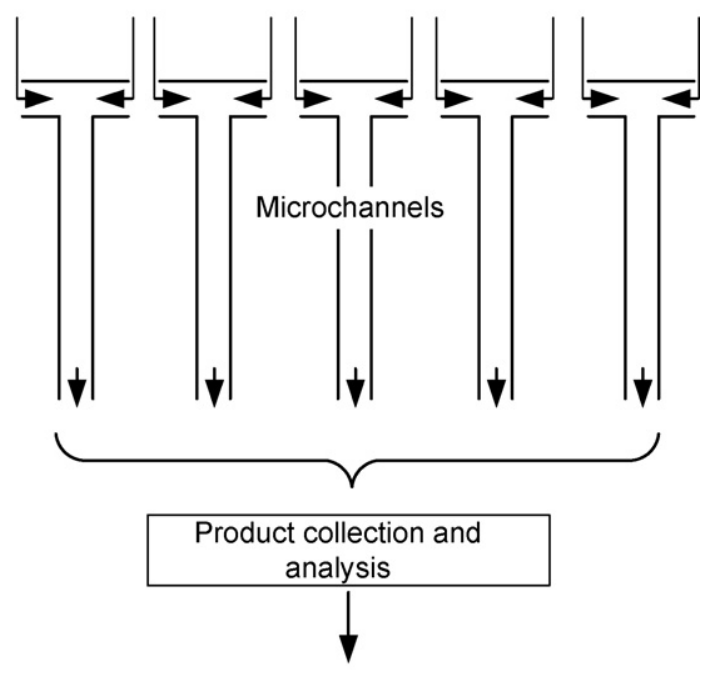

(b)

Fig. 1. Numbering-up concept for two-phase MSR. (a) Internal numbering-up and (b) external numbering-up. 


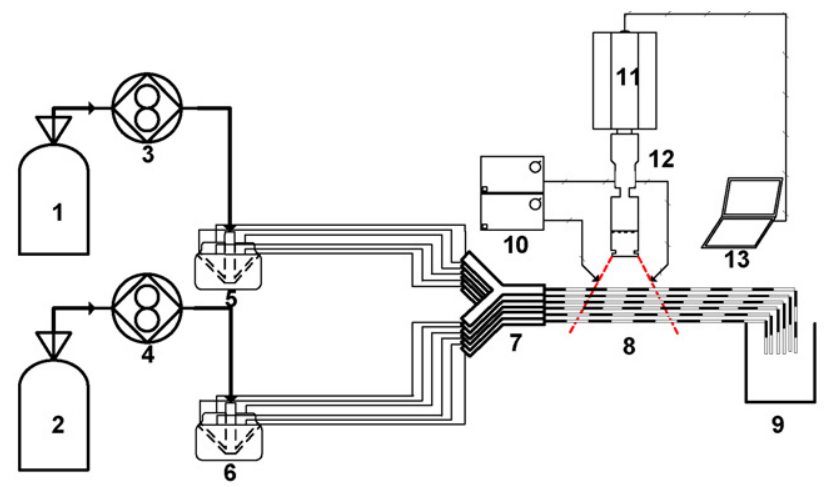

(a) (1) reservoir ${ }_{\mathrm{aq}}$; (2) reservoir $_{\mathrm{or}} ;$ (3) pump $_{\mathrm{aq}}$; (4) pump $_{\mathrm{or}}$; (5) distributor ${ }_{\mathrm{aq}} ;$; (6)

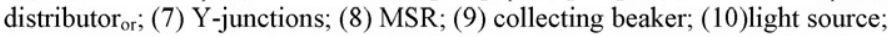
(11) high speed camera; (12) optical microscope; (13) laptop.

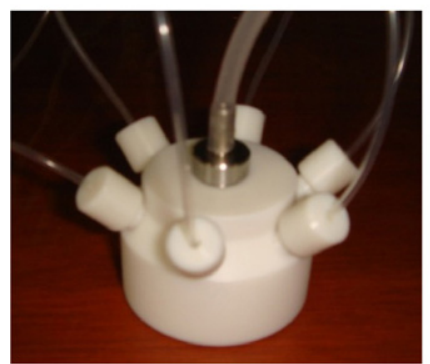

(b)

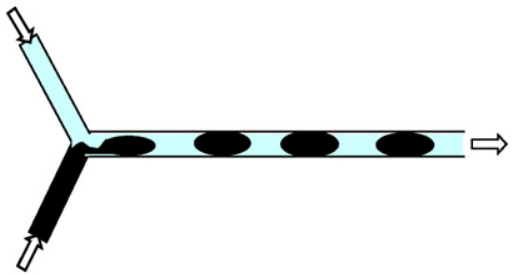

(c)

Fig. 2. Experimental set-up. (a) Schematic representation, (b) distributor and (c) schematics of Y-junction.

\section{Experimental}

The experimental set-up developed for six parallel MSR is shown in Fig. 2a. It consists of two single-phase flow distributors, six two-phase Y-shape mixing elements, six PTFE capillaries attached downstream acting as MSR and a high speed imaging system located halfway along the length of the capillaries. The distributor is made of Teflon ${ }^{\circledR}$ (Fig. 2b) with an inlet internal diameter of $3 \mathrm{~mm}$ and outlet internal diameter of $1 \mathrm{~mm}$, each. All the outlets are inclined at an angle of $45^{\circ}$ with the inlet. A pair of outlet streams, one from each of the two distributors, is connected to a symmetric $120^{\circ}$ Y-shape mixing element (schematically shown in Fig. 2c) having equal internal diameter of $0.5 \mathrm{~mm}$ for all of its openings. The high speed image recording and analysis system consists of a high-speed video camera attached to the microscope, light sources to illuminate the area, and a laptop to acquire the images.

Two immiscible liquids, aqueous and organic, from respective reservoirs were introduced by gear pumps to flow distributors where each inlet stream is split into six outlet streams. The experimental parameter and operating conditions used in this work are given in Table 1 . The distributor does not have any active flow regulation parts, but achieves flow equipartition due to the symmetry of the element and the pressure drop generated in the downstream Yjunction mixing element. At the outlets, the samples were collected and analyzed.

Initially, two-phase flow experiments were conducted to study the flow regimes in the numbered-up set-up for both equal and unequal flow rates of the two phases using high speed imaging system. The idea was to define a stable operating window for the slug-flow regime to carry out mass transfer experiments. A non-reacting system, water (colored with brilliant blue dye)cyclohexane, was chosen for this work. In order to estimate the flow deviation in slug-flow, the outlet flow volume of each phase from each of the six capillaries was measured.
To characterize the mass transfer, the laboratory benchmark system of kerosene (+acetic acid)-water was chosen which has been tested before for single capillary [4]. Experiments were performed using aqueous phase as water with or without surfactant. A cationic surfactant, CTAB (cetyl trimethyl ammonium bromide), in various concentrations $(0.1$ and $0.2 \mathrm{~g} / \mathrm{L}$ which is below the critical micelle concentration (CMC), $0.33 \mathrm{~g} / \mathrm{L}$ ) was used. The interfacial tensions measured using ring method (Krüss tensiometer K6, Krüss $\mathrm{GmbH}$, Germany) for different surfactant concentrations are given in Table 2. Measurements were taken for varying flow rates of both phases, keeping the flow ratio equal to one, at a constant capillary length (i.e. varying residence time). Quantitative analysis of the acetic acid transferred to aqueous phase was done using HPLC technique. Three samples from each set of experiments were analyzed and the mean value was recorded.

Table 1

Experimental parameters and operating conditions used.

\begin{tabular}{|c|c|}
\hline Parameter & Chemicals/value \\
\hline \multicolumn{2}{|l|}{ Hydrodynamics } \\
\hline System & Water-cyclohexane \\
\hline Capillary material & Polytetrafluoroethylene (PTFE) \\
\hline Total flow rate $[\mathrm{mL} / \mathrm{h}]$ & $60-600$ \\
\hline No. of capillaries & 6 \\
\hline Two-phase capillary & 1000 \\
\hline \multicolumn{2}{|l|}{ length [mm] } \\
\hline $\begin{array}{l}\text { Capillary and Y-junction } \\
\text { internal diameter [mm] }\end{array}$ & 0.5 \\
\hline \multicolumn{2}{|l|}{ Mass transfer } \\
\hline System & Kerosene-acetic acid-water \\
\hline $\begin{array}{l}\text { Initial concentration of } \\
\text { acetic acid in kerosene }[\mathrm{g} / \mathrm{L}]\end{array}$ & 2 \\
\hline $\begin{array}{l}\text { CTAB surfactant } \\
\text { concentration in aqueous } \\
\text { phase }[\mathrm{g} / \mathrm{L}]\end{array}$ & $0,0.1,0.2$ \\
\hline
\end{tabular}


Table 2

Interfacial tensions $(\gamma)$ of systems used.

\begin{tabular}{ll}
\hline System & $\gamma(\mathrm{mN} / \mathrm{m})$ \\
\hline Kerosene-distilled water & 47 \\
Kerosene-0.1 g/L CTAB in water & 18 \\
Kerosene-0.2 g/L CTAB in water & 11 \\
\hline
\end{tabular}

Two parameters, volumetric mass transfer coefficient and extraction efficiency, were evaluated to quantify the mass transfer phenomenon. The overall volumetric mass transfer coefficient can be calculated from the following equation [5]:

$k_{\mathrm{L}} a=\frac{C_{\text {out }}-C_{\text {in }}}{\tau \cdot\left(\left(\left(C_{\text {in }}^{*}-C_{\text {in }}\right)-\left(C_{\text {out }}^{*}-C_{\text {out }}\right)\right) /\left(\ln \left[\left(C_{\text {in }}^{*}-C_{\text {in }}\right) /\left(C_{\text {out }}^{*}-C_{\text {out }}\right)\right]\right)\right)}$

where $C_{\text {in }}, C_{\text {out }}, C_{\text {in }}^{*}, C_{\text {out }}^{*}$ are the inlet, outlet, inlet equilibrium and outlet equilibrium concentrations of the acetic acid in the aqueous phase, respectively. The residence time $\tau$ in each capillary can be calculated dividing the volume of capillary by total volumetric flow rate.

Extraction efficiency $(E)$ is the ratio of the amount of material transferred to the maximum amount transferable. For a solute transferring from one phase to another, the extraction efficiency can be written as follows [4]:

$E=\frac{C_{\text {out }}-C_{\text {in }}}{C_{\text {out }}^{*}-C_{\text {in }}}$

The extraction efficiency of the MSR not only dependent on the mass transfer coefficient but also on the residence time of fluids. Therefore, the mass transfer capacity of the reactor, is defined analogous to first Damköhler number as given in the following:

$D a^{\prime}=k_{\mathrm{L}} a \cdot \tau$

The performance of flow distributor was investigated using CFD simulations. Pressure drop is an important parameter to be characterized for the flow distributor. Therefore, steady state CFD simulations were conducted using commercial finite volume based software FLUENT at varying flow rates from 60 to $600 \mathrm{~mL} / \mathrm{h}$. The contour diagrams for the pressure drop and velocity magnitude are shown in Fig. 3b and c, respectively. The pressure drop between the inlet and outlet of the distributor was calculated for different flow rates from 60 to $600 \mathrm{~mL} / \mathrm{h}$. To analyze the effect of vertical angle between outlet arms and the inlet $(\theta)$ on the pressure drop, simulations were carried out for different $\theta$ values.

\section{Results and discussion}

\subsection{Flow distribution and flow regimes}

The CFD simulations for hydrodynamics of flow distributor are shown in Fig. 3. It shows the equipartition of liquid in all channels at very low pressure drop. The pressure drop increases with increasing flow rate and does not show an effect of angle $\theta$ (Fig. 3d). In comparison, the pressure drop due to a two-phase mixing element and a two-phase slug-flow capillary is very high (for example for a Y-junction and capillary with diameter $0.5 \mathrm{~mm}$, pressure drop in Y-junction $\sim 9-34 \mathrm{kPa}$, slug-flow $\sim 20-38 \mathrm{kPa} / \mathrm{m}$ for a velocity range of $7-70 \mathrm{~mm} / \mathrm{s}$ [3]).

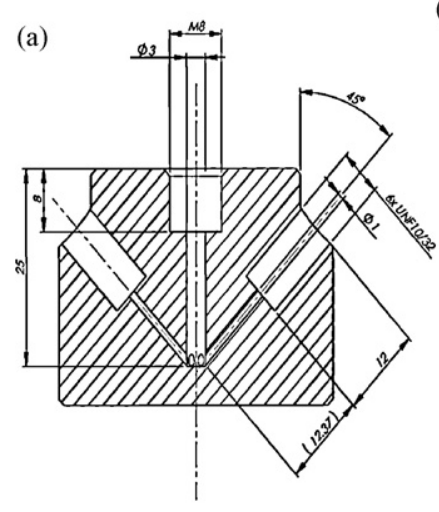

(b)

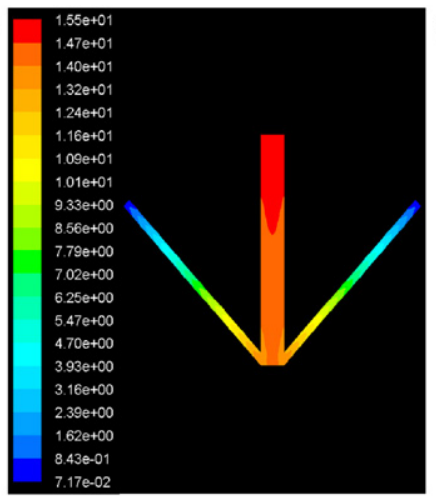

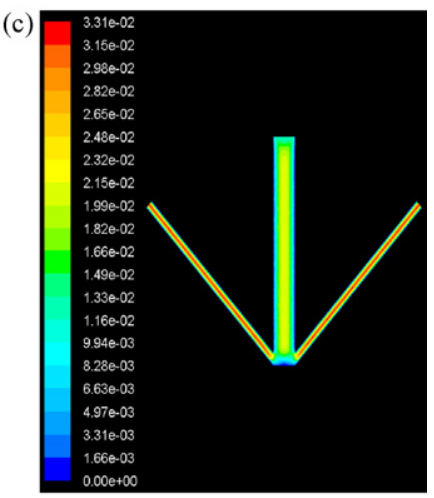

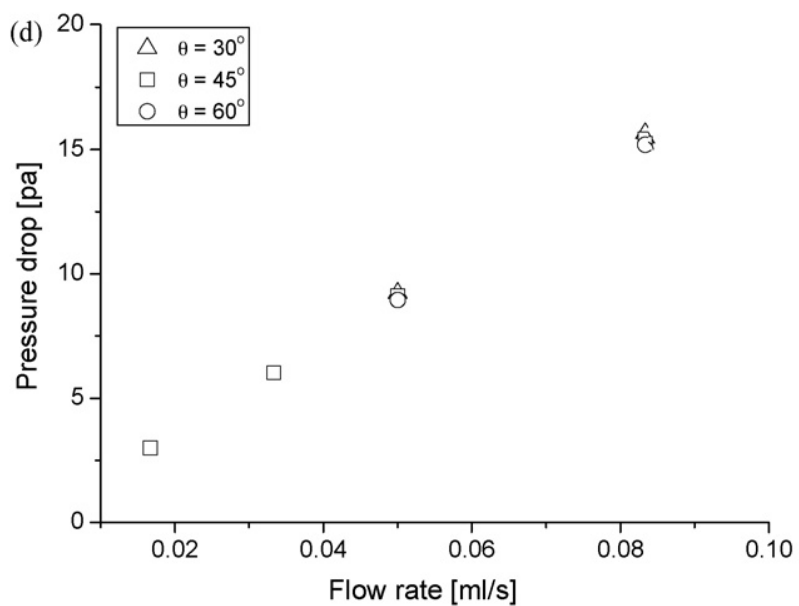

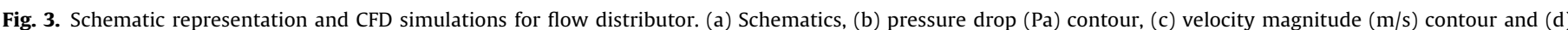
pressure drop in the distributor as a function of flow rate for different $\theta$. 


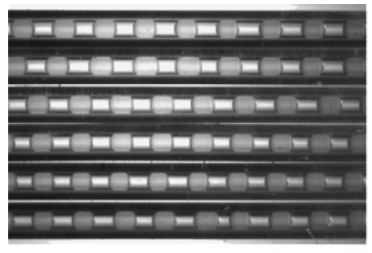

(a) Slug flow

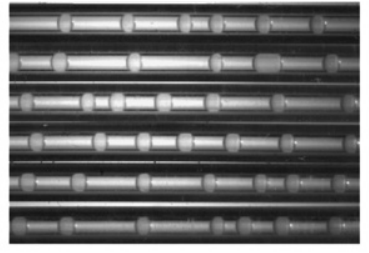

(b) Drop flow

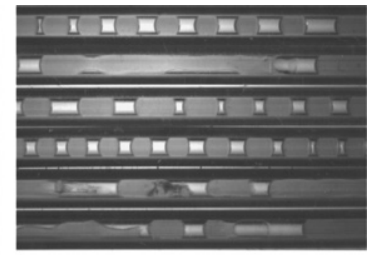

(c) Deformed interface flow

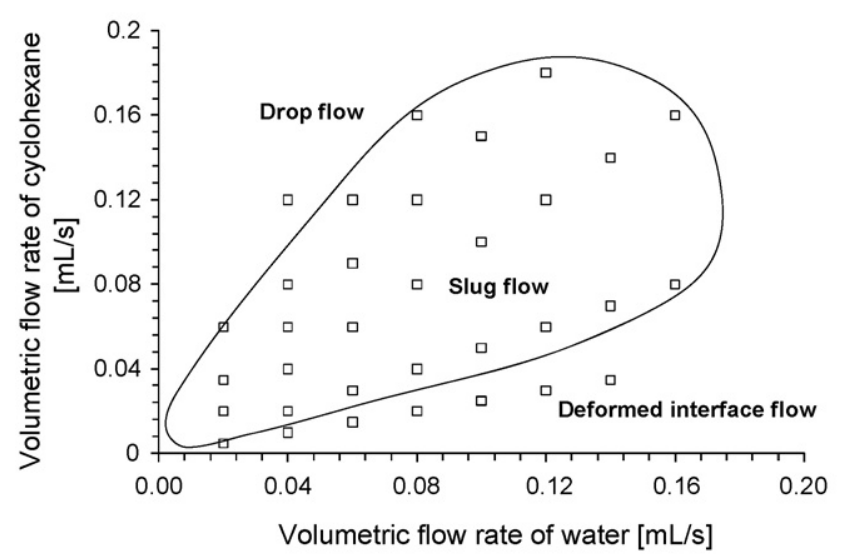

(d) Slug flow operating window

Fig. 4. Observed flow regimes in six parallel capillary MSR.

Experiments were carried out to study the flow distribution of single phase flow and observed almost ideal flow partition (relative standard deviation (RSD), 1-4\%). When experiments were conducted to characterize two-phase liquid-liquid flow in the numbered-up setup at varying flow rates and flow ratios using water-cyclohexane system, typically three distinct flow regimes, namely well-defined slug-flow, drop flow and deformed interface flow, were observed as shown in Fig. 4. Water forms convex shaped slug while cyclohexane exhibits a concave geometry, as would be expected with the hydrophobic PTFE wall material. The exact form of the slug depends on the volumetric flow rates and inlet flow ratio.

The flow regime map is shown in Fig. 4d. As can be seen, stable slug-flow was observed for equal or nearly equal flow rates of both fluids in a range of upto $0.028 \mathrm{~mL} / \mathrm{s}$ per capillary which corresponds to the value reported in the literature $(\sim 0.02 \mathrm{~mL} / \mathrm{s}$ [3] $)$. The flow at unequal flow rates of both phases revealed that transition of the slug-flow to drop flow occurs in the range of cyclohexane/water flow ratio of 2-3 and the transition to deformed interface flow occurs at water/cyclohexane flow ratio of about 2-3. Thus, from these observations, a conclusion could be made that scaleout approach of capillary microchannels does not alter the flow behavior.

Volumetric measurements of the outlet flow of both phases in each capillary were done for varying flow rates and an average outlet flow ratio, RSD of the distribution were determined for slug-flow regime. Fig. 5 shows the deviation of flow ratio in each capillary outlet for equal flow rates of both phases. As can be seen, the average deviation is very low (RSD-1-4\%) confirming uniform slug-flow in all capillaries. Thus, without using any active flow distribution control elements, an equal flow distribution of both phases in each capillary with numbered up setup was achieved.

For the well-defined slug-flow regime, interfacial area per unit slug volume was investigated from the experimental snapshots by measuring the slug length. It has been previously reported that in water-cyclohexane system, organic wall film is present [3]. So, the convex water slugs are surrounded by organic phase and interfacial area is just the total surface area of the water slugs. To measure it, we assume that water slugs have spherical caps and both ends of the slug are symmetrical.

As volumetric flow rate increases, the slug size decreases due to rapid penetration of one phase into another. One thus achieves higher interfacial area per unit volume. The range of mean interfacial area per capillary varies from 4700 to $5100 \mathrm{~m}^{2} / \mathrm{m}^{3}$ (with the assumption of organic wall film) which corresponds to the previous reported values in single capillary [3]. This suggests that numbered up setup also provides the same performance with respect to interfacial area in each capillary as is the single capillary. The interfacial area for a mechanically agitated tank reactor is as high as $500 \mathrm{~m}^{2} / \mathrm{m}^{3}$. It means that one order of magnitude increase in interfacial area was achieved in the present setup.

\subsection{Mass transfer coefficient and extraction efficiency}

The volumetric mass transfer coefficients are plotted as a function of slug-flow velocity for the case of pure water and CTAB in

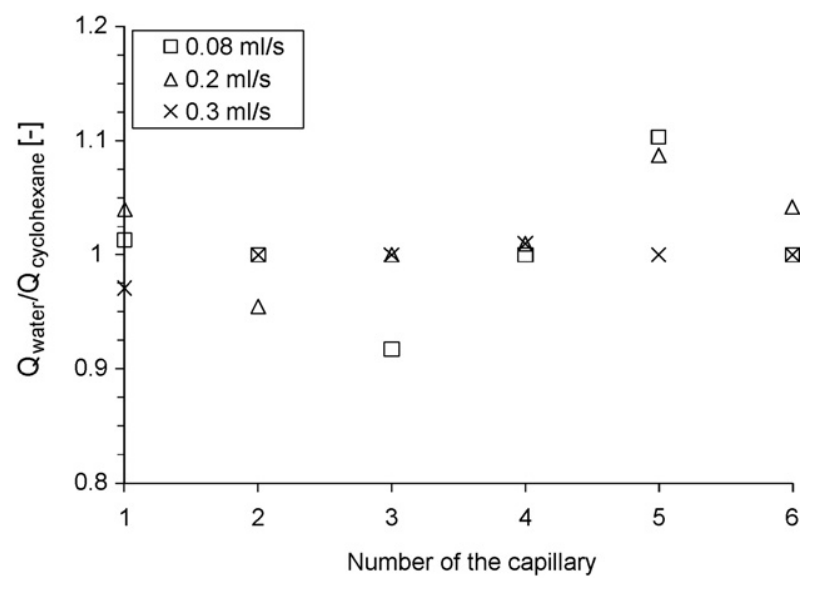

Fig. 5. Flow distribution in six parallel capillaries for different total flow rates for equal flow rates of water and cyclohexane. 


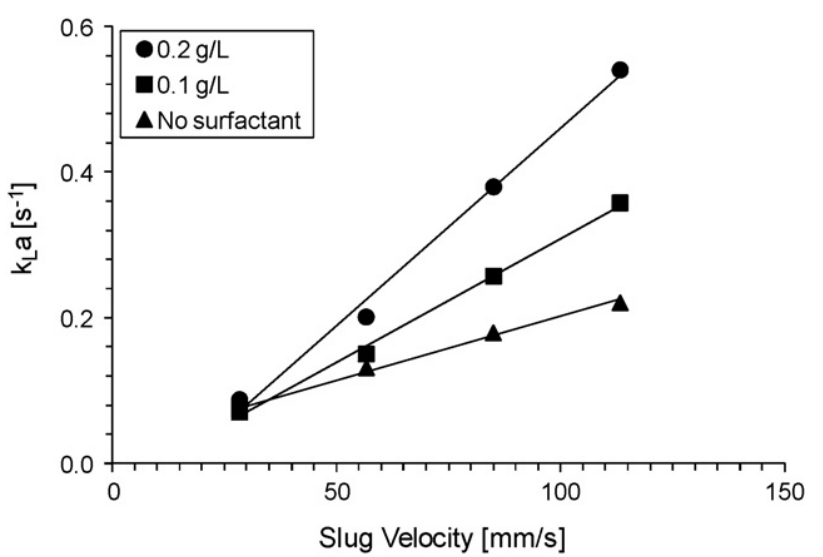

Fig. 6. Volumetric mass transfer coefficient as a function of slug-flow velocity for various surfactant concentrations.

water in Fig. 6 and the experimental flow snapshots are depicted in Fig. 7. As can be seen, with the increase in slug-flow (linear) velocity, the mass transfer coefficients increase over the entire slugflow regime. This is due to increase in interfacial area and intense internal circulations within the slug, which in turn, enhances the convective mass transfer. The former effect is due to the decrease in the slug size with flow velocity and latter effect is because of higher shear rate with higher flow velocity. Though the experimental snapshots of 'clean' aqueous solution without any surfactant do not show the slug size difference with flow velocity, the microscopic analysis reveals the difference.

In presence of the surfactant, mass transfer coefficient values are higher compared to the 'clean' aqueous solution. Though there is no significant difference between the slug size for with and without surfactant at lower flow velocities, the difference in mass transfer rates is attributed to change in the interfacial tension. However, at higher flow velocities, the hydrodynamics of two-phase system change significantly due to surfactant. At the flow velocity of $113.2 \mathrm{~mm} / \mathrm{s}$, without CTAB a well-defined slug-flow is observed while with CTAB drop flow is formed (Fig. 7). The interfacial area is increased by $30 \%$ for increase in flow velocity from 84.84 to $113.2 \mathrm{~mm} / \mathrm{s}$. Though the interfacial area shows sudden rise, the rate of change of mass transfer coefficient per unit flow velocity remains constant. The volumetric mass transfer coefficients found in this work for MSR are much greater (at least one order of magnitude) than those of conventional contactors [4] (for example, $k_{\mathrm{L}} a$ in conventional stirred tank is as high as $0.02 \mathrm{~L} / \mathrm{s}$ ).

The extraction efficiency for different surfactant concentrations is plotted in Fig. 8. Without surfactant, $E$ decreases as slug-flow

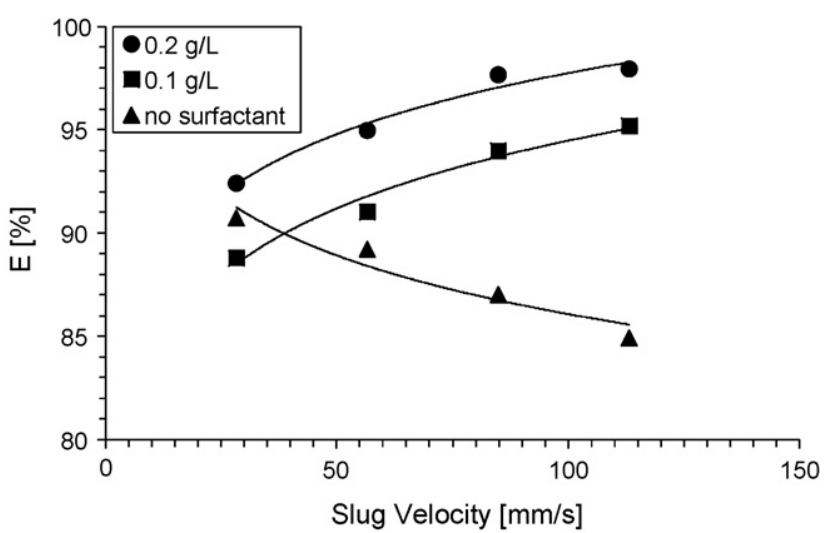

Fig. 8. Extraction efficiency of kerosene-acetic acid-water system in MSR (capillary $\mathrm{ID}=0.5 \mathrm{~mm}$, Y-junction ID $=0.5 \mathrm{~mm}$ and two-phase capillary length $=1000 \mathrm{~mm}$ ).

velocity increases because the decrease in contact time. However, the presence of surfactant increased the extraction efficiency with flow velocity for a given capillary length. It can be explained in terms of very high rates of change of mass transfer coefficients with respect to flow velocity in the presence of surfactant compared to clean solution as shown in Fig. 6 .

The results obtained in the present work were compared with the experimental data for single capillary from Kashid et al. [4]. Since the two-phase capillary length used in the present work is different, the extraction efficiency instead of the volumetric mass transfer coefficients was compared. The extraction efficiency as a function of $D a^{\prime}$ is shown in Fig. 9 with general trend to increase with increase in $D a^{\prime}$. The high values of $E$ and $D a^{\prime}$ in numbered-up setup compared to single capillary are due to very high volumetric mass transfer coefficient in the presence of surfactant. The results for both single capillary and numbered-up setup show a very little difference. The behavior of extraction efficiency in percent with $D a^{\prime}$ can be expressed as

$E=100\left(1-e^{-D a^{\prime}}\right)$

The above equation is similar to the first order reaction kinetics where conversion (here extraction efficiency) is expressed as a function of first Damköhler number (here $D a^{\prime}$ ).

As mentioned before, the effect of surfactant on the liquid-liquid extraction has been studied in the literature. The results of Blokker [13], Davies and Wiggill [14], Mudge and Heideger [15], indicated decreased mass transfer in the presence of surfactants in ternary systems. This effect was attributed either to a mechanical resistance (barrier effect) or the dampening of Marangoni convection at the interface caused by the formation of a rigid interfacial film. The

Without surfactant

Surfactant concentration $0.2 \mathrm{~g} / \mathrm{L}$

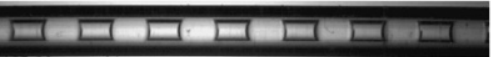

(a) $28.28 \mathrm{~mm} / \mathrm{s}$
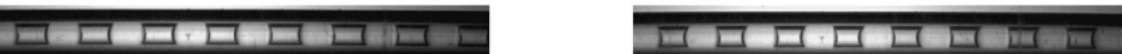

(b) $56.56 \mathrm{~mm} / \mathrm{s}$

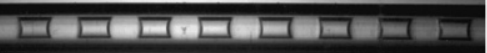

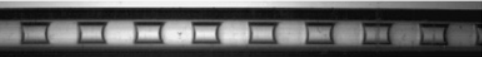

(c) $84.84 \mathrm{~mm} / \mathrm{s}$

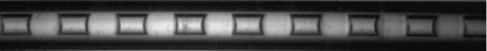

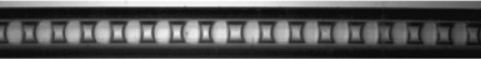

(d) $113.2 \mathrm{~mm} / \mathrm{s}$

Fig. 7. Effect of flow velocity and surfactant concentration on the slug size for kerosene-acetic acid-water. 


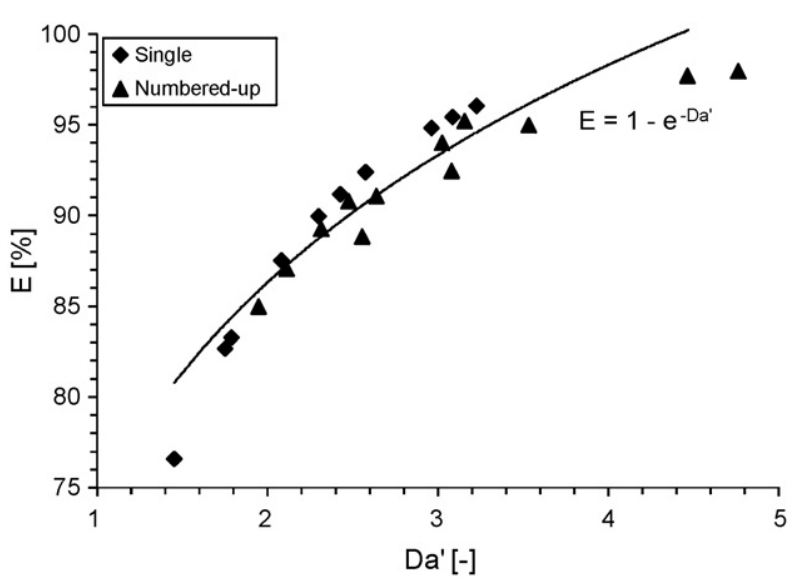

Fig. 9. Comparison of extraction efficiency of single and numbered-up capillaries (capillary ID $=0.5 \mathrm{~mm}$, Y-junction ID $=0.5 \mathrm{~mm}$ ).

presence of surfactants was, therefore, considered disadvantageous for mass transfer. However, recent studies have shown increment in mass transfer rates due to the occurrence of Marangoni convection at the interface $[12,16]$.

In the liquid-liquid slug-flow, when the slug moves along the length of the capillary in a straight channel, highly regular internal circulations are developed as shown in Fig. 10a. This hypothesis is supported by computational fluid dynamics simulations [17] and particle image velocimetry measurements $[17,18]$. Besides internal circulations, the liquid in some part of the slug is stagnant where the mass transfer is due to diffusion only. However, in the presence of surfactant, Marangoni effects are strong and the internal circulation is hindered due to local convection patterns initiated by the movement of the interface. The schematic representation of hindered internal circulations in a slug of liquid-liquid slugflow is shown in Fig. 10b. Such a change in internal circulation due to strong Marangoni effect was postulated by Wegener et al. [16] for dispersed liquid-liquid systems. The fluid within the slug is well-mixed and generates high concentration gradient across the biphasic interface and therefore it enhances the rate of diffusive penetration across the interface. Thus, the presence of surfactant in the aqueous phase can increase the mass transfer rates.

\subsection{Correlation of the data}

No empirical or semi-empirical model is available to investigate the mass transfer coefficient in the MSR. Thus, dimensional analysis was employed to obtain a relationship between the measured quantities. It is assumed that the following independent variables should play roles in the extraction processes:

$k_{\mathrm{L}} a=f\left(u_{\mathrm{TP}}, d_{\mathrm{c}}, L_{\text {slug }}, L, \rho_{1}, \rho_{2}, \mu_{1}, \mu_{2}, \gamma\right)$

where $u_{\mathrm{TP}}, d_{\mathrm{c}}, L_{\text {slug }}, L, \rho, \mu$ are the slug velocity, channel diameter, length of the slug(cap-to-cap) and length of two-phase capillary, density, dynamic viscosity, respectively. The subscripts 1 and 2 denote kerosene and water phases. Eq. (5) was transformed into the following groups by using the Buckingham Pi method:

$$
\begin{aligned}
D a^{\prime}= & k_{\mathrm{L}} a \cdot \tau=\left(\frac{k_{\mathrm{L}} a \cdot L}{u_{\mathrm{TP}}}\right) \\
& =a \cdot(\mathrm{Ca})^{b} \cdot(\operatorname{Re})^{c} \cdot\left(\frac{d_{\mathrm{c}}}{L}\right)^{d} \cdot\left(\frac{L_{\mathrm{slug}}}{d_{\mathrm{c}}}\right)^{e} \cdot\left(\frac{\rho_{\mathrm{D}}}{\rho_{\mathrm{C}}}\right)^{f} \cdot\left(\frac{\mu_{\mathrm{D}}}{\mu_{\mathrm{C}}}\right)^{g}
\end{aligned}
$$

where $C a$ is capillary number $\left(u_{\mathrm{TP}} \mu_{\mathrm{M}} / \gamma\right)$ and Re is the Reynolds number $\left(\rho_{\mathrm{M}} u_{\mathrm{TP}} d_{\mathrm{c}} / \mu_{\mathrm{M}}\right)$. Here $\mathrm{M}$ refers to the mixture properties which are calculated using phase fraction $(\varphi)$ by the following expressions:

$$
\begin{aligned}
\rho_{\mathrm{M}} & =\left(\frac{\varphi_{1}}{\rho_{1}}+\frac{1-\varphi_{1}}{\rho_{2}}\right)^{-1} ; \quad \mu_{\mathrm{M}}=\left(\frac{\varphi_{1}}{\mu_{1}}+\frac{1-\varphi_{1}}{\mu_{2}}\right)^{-1} ; \\
\varphi_{1} & =\left(\frac{Q_{1}}{Q_{1}+Q_{2}}\right)
\end{aligned}
$$

where $Q$ is the volumetric flow rate. The exponents of last two terms in Eq. (6) are very small and therefore, they can be combined with constant ' $a$ '. Besides, it was observed that $L_{\text {slug }} / d$ shows about $10 \%$ of difference with flow velocity, and therefore, it was also combined with $a$ in order to reduce number of fitting parameters and to obtain good fitting for $\mathrm{Ca}$ and Re. Thus, the following equation is formed with a new constant ' $a$ ":

$D a^{\prime}=k_{\mathrm{L}} a \cdot \tau=\left(\frac{k_{\mathrm{L}} a \cdot L}{u_{\mathrm{TP}}}\right)=a^{\prime} \cdot(C a)^{b} \cdot(R e)^{c} \cdot\left(\frac{d_{\mathrm{c}}}{L}\right)^{d}$

The constant $a^{\prime}$ and the exponents $b-d$ are adjustable parameters that were determined by fitting experimental data to the correlation.

This equation was applied to the data obtained from single capillary experiments without surfactant as well as from previously reported literature [4] and the correlation obtained is given as

$D a^{\prime}=1 \cdot(C a)^{-0.09} \cdot(R e)^{-0.09} \cdot\left(\frac{d_{\mathrm{c}}}{L}\right)^{-0.1}$

Similarly, correlation for Damköhler number from the data obtained in numbered-up without surfactant is given as

$D a^{\prime}=0.88 \cdot(C a)^{-0.09} \cdot(R e)^{-0.09} \cdot\left(\frac{d_{\mathrm{c}}}{L}\right)^{-0.1}$

Thus, almost similar $D a^{\prime}$ was achieved in numbered-up setup compared to single capillary with a deviation in acceptable range- $a^{\prime}$ equals 0.88 instead of 1 .

Further, a correlation was fitted for data obtained in the presence of surfactant. If the density and viscosity are assumed to be constant, the variation in mass transfer rates with surfactant concentration is attributed to the change in interfacial tension and intensity of internal circulations within the slugs due to flow velocity. As a result, constant $a^{\prime}$ and exponents b-c were changed while exponent was kept the same and following equation was obtained:

$$
\begin{aligned}
& \text { Surfactant }=0.1 \mathrm{~g} / \mathrm{L}: D a^{\prime}=0.9 \cdot(\mathrm{Ca})^{0.03} \cdot(R e)^{0.18} \cdot\left(\frac{d_{\mathrm{c}}}{L}\right)^{-0.1} \\
& \text { Surfactant }=0.2 \mathrm{~g} / \mathrm{L}: D a^{\prime}=0.9 \cdot(\mathrm{Ca})^{0.08} \cdot(R e)^{0.36} \cdot\left(\frac{d_{\mathrm{c}}}{L}\right)^{-0.1}
\end{aligned}
$$

The coefficients $a^{\prime}, b$ and $c$ in the above equation are changed showing that both capillary number and Reynolds number play an important role in the presence of surfactant. It is important to note that the above equation is valid below the velocity of $85 \mathrm{~mm} / \mathrm{s}$ in a $0.5 \mathrm{~mm}$ ID capillary because further increase in flow velocity results in drop (slug with length less than capillary diameter) flow. In the absence of surfactant, the exponents $b$ and c have negative values while with addition of surfactant they become positive and increase with increase in the surfactant concentration. This is due to change in interfacial tension resulting in different flow patterns within the slug as discussed in the above section. Finally, the correlated data are plotted with experimental results for all cases of single capillary, numbered-up capillaries with and without surfactant in Fig. 11. The correlated data are $95 \%$ agreement with experimental results. Thus, this suggests that the mass transfer performance of liquid-liquid slug-flow can be correlated and be used for a priori prediction of mass transfer rates.

Since the change in $k_{\mathrm{L}} a$ in the presence of surfactant is due to interfacial tension $(\gamma)$, the values of two parameters are correlated 

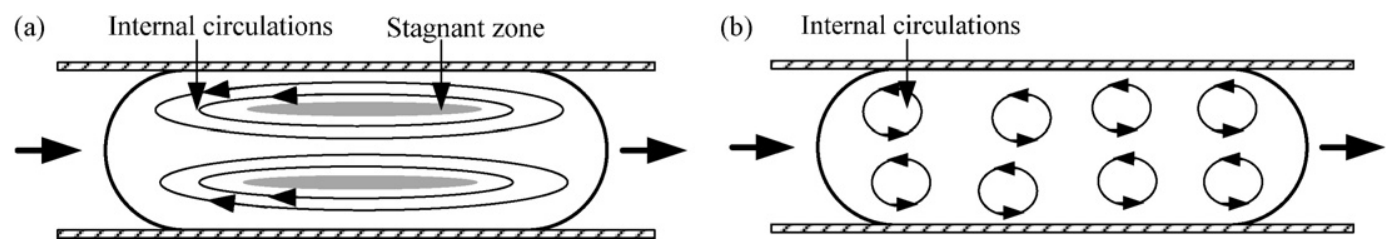

Fig. 10. Internal circulations within the aqueous slug. (a) Without surfactant and (b) with surfactant.

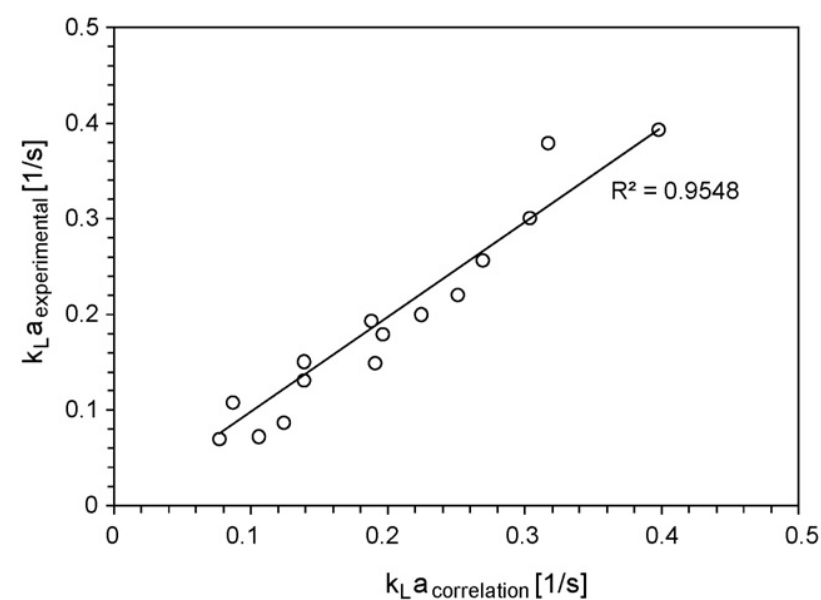

Fig. 11. Parity plot showing agreement between experimental and correlated data for single, numbered-up and numbered-up capillaries with surfactant.

and following relation was obtained:

$$
\begin{aligned}
k_{\mathrm{L}} a_{0.2 \mathrm{~g} / \mathrm{L}} & \approx 1.6 k_{\mathrm{L}} a_{0.1 \mathrm{~g} / \mathrm{L}} \\
\approx & \frac{\gamma_{0.1 \mathrm{~g} / \mathrm{L}}}{\gamma_{0.2 \mathrm{~g} / \mathrm{L}}} k_{\mathrm{L}} a_{0.1 \mathrm{~g} / \mathrm{L}}
\end{aligned}
$$

where, $k_{\mathrm{L}} a_{\mathrm{i}}$ and $\gamma_{\mathrm{i}}$ are the volumetric mass transfer coefficients and interfacial tension at surfactant concentration $i$, respectively.

\section{Conclusions}

Flow distribution and mass transfer performance of the liquid-liquid system was studied in the numbered-up setup for six capillaries. The flow regime experiments proved that not only the operating window of single capillary was preserved for the slug-flow but also the total throughput was increased sixfold. The mass transfer performance was investigated for two cases: without and with surfactant. In absence of surfactant, mass transfer rates were increased with rise in slug-flow velocity and followed the same trend as reported for single capillary. Further increase in mass transfer rates were observed in the presence of surfactant due to enhanced internal circulations within the slug resulting from strong interfacial movement. Finally, the mass transfer rates correlated as a function of Reynolds number, capillary number, diameter and length of the capillary showed very good agreement (95\%) with experimental results. Thus, the potential of surfactants to control mass transfer phenomenon and the applicability of numbering-up technique to increase the performance proportionally strengthen the fact that the benefits of MSR and throughput of conventional reactors can be achieved in micro-set-up.

\section{References}

[1] G. Dummann, U. Quittmann, L. Groschel, D.W. Agar, O. Worz, K. Morgenschweis, The capillary-microreactor: a new reactor concept for the intensification of heat and mass transfer in liquid-liquid reactions, Catal. Today 79 (1-4) (2003) 433-439.

[2] J.R. Burns, C. Ramshaw, The intensification of rapid reactions in multiphase systems using slug flow in capillaries, Lab Chip 1 (1) (2001) 10-15.

[3] M.N. Kashid, D.W. Agar, Hydrodynamics of liquid-liquid slug flow capillary microreactor: flow regimes, slug size and pressure drop, Chem. Eng.J. 131 (1-3) (2007) 1-13.

[4] M.N. Kashid, Y.M. Harshe, D.W. Agar, Liquid-liquid slug flow in a capillary: an alternative to suspended drop or film contactors, Ind. Eng. Chem. Res. 46 (25) (2007) 8420-8430.

[5] Y. Zhao, G. Chen, Q. Yuan, Liquid-liquid two-phase mass transfer in the Tjunction microchannels, AIChE J. 53 (12) (2007) 3042-3053.

[6] A.L. Dessimoz, L. Cavin, A. Renken, L. Kiwi-Minsker, Liquid-liquid two-phase flow patterns and mass transfer characteristics in rectangular glass microreactors, Chem. Eng. Sci. 63 (16) (2008) 4035-4044.

[7] W. Ehrfeld, V. Hessel, H. Löwe, Microreactors, Wiley-VCH Verlag GmbH, Weinheim, 2000.

[8] M.N. Kashid, L. Kiwi-Minsker, Microstructured reactors for multiphase reactions: state of the art, Ind. Eng. Chem. Res. 48 (14) (2009) 6465-6485.

[9] J.R. Burns, C. Ramshaw, A microreactor for the nitration of benzene and toluene, Chem. Eng. Commun. 189 (12) (2002) 1611-1628.

[10] R. Schenk, V. Hessel, C. Hofmann, J. Kiss, H. LoÎ we, A. Ziogas, Numbering-up of micro devices: a first liquid-flow splitting unit, Chem. Eng. J. 101 (1-3) (2004) 421-429.

[11] T. Iwasaki, N. Kawano, J.I. Yoshida, Radical polymerization using microflow system: numbering-up of microreactors and continuous operation, Org. Process. Res. Dev. 10 (6) (2006) 1126-1131.

[12] D. Agble, M.A. Mendes-Tatsis, The effect of surfactants on interfacial mass transfer in binary liquid-liquid systems, Int. J. Heat Mass Trans. 43 (6) (2000) 1025-1034.

[13] P.C. Blokker, On mass transfer across liquid-liquid interfaces in systems with and without surface active agents, in: Second International Congress on Surface Activity, 1957, pp. 503-511.

[14] J.T. Davies, J.B. Wiggill, Diffusion across the oil/water interface, Proc. R. Soc (London) (A255) (1960) 277-291.

[15] L.K. Mudge, W.J. Heideger, Effect of surface active agents on liquid-liquid mass transfer rates, AIChE J. 16 (4) (1970) 602-608.

[16] M. Wegener, M. Fevre, A.R. Paschedag, M. Kraume, Impact of Marangoni instabilities on the fluid dynamic behaviour of organic droplets, Int. J. Heat Mass Trans. 52 (11-12) (2009) 2543-2551.

[17] M.N. Kashid, I. Gerlach, S. Goetz, J. Franzke, J.F. Acker, F. Platte, D.W. Agar, S Turek, Internal circulation within the liquid slugs of a liquid-liquid slug-flow capillary microreactor, Ind. Eng. Chem. Res. 44 (14) (2005) 5003-5010.

[18] D. Malsch, M. Kielpinski, R. Merthan, J. Albert, G. Mayer, J.M. Köhler, H. Süße, M. Stahl, T. Henkel, $\mu$ PIV-Analysis of Taylor flow in micro channels, Chem. Eng. J. 135 (Suppl. 1) (2007) S166-S172. 